\title{
El análisis y la formulación de estrategias como elemento de la competitividad de micro, pequeñas y medianas empresas (mipymes) en el área metropolitana de Bucaramanga*
}

Strategic analysis and practice as a competitive element of micro, small and medium-size enterprises in the Bucaramanga metropolitan area

Ancelmo José Vega Armenta** Artículo de investigación cientifica y tecnológica

\section{Resumen}

El análisis y la formulación de estrategias en micro, pequeñas y medianas empresas (mipymes) en los países de menor desarrollo industrial se ha adelantado principalmente como un componente más de la gestión administrativa y tecnológica, focalizada en el entorno externo empresarial y mediante el empleo de modelos originalmente formulados para grandes organizaciones multifuncionales. Con este tipo de abordaje, infortunadamente se ha subvalorado o desconocido la relevancia de elementos que, como la flexibilidad de este tipo de organizaciones empresariales, pueden constituirse en fuentes de ventaja sostenibles.

* Este documento ha sido realizado como parte del proyecto "Diseño de una metodología de análisis que considere variables organizacionales internas y del entorno para llevar a cabo mediciones sistemáticas de la competitividad organizacional y sectorial de las Mipymes del área metropolitana de Bucaramanga", que se elabora de manera conjunta entre el grupo de investigación en Economía Social y Desarrollo Empresarial de la Maestría en Administración de Empresas de la Universidad Santo Tomás, sede Bucaramanga y el grupo GRIES de la Maestría en Ciencias Económicas de la Universidad Santo Tomás, sede Bogotá. Correo electrónico: ajvegar@gmail.com

** Docente, maestría en Ciencias Económicas, Universidad Santo Tomás, Bogotá. Ingeniero Mecánico, magíster en Ciencias Económicas, Ph.D (c). 
En este artículo se presenta un modelo de análisis y práctica estratégica en la perspectiva de la teoría basada en los recursos; los desarrollos de Grant, 2010; Rangone, 1999 y Hall, 1992, adecuados a las características de mipymes. La característica más relevante del modelo es la facilidad para su aplicación por parte de los propietarios/ adminitradores de estas organizaciones. Con esto se desea contribuir a una mejor comprensión del funcionamiento de las mipymes en nuestro medio.

Palabras clave: Teoria basada en los recursos, comportamiento estratégico, planeación estratégica, comportamiento empresarial.

\section{Abstract}

Strategic analysis and practice in micro, small and medium-size enterprises in less development countries has been put forward mainly as another component of the

\section{Introducción}

El análisis y la práctica estratégica en las organizaciones empresariales como parte de los retos competitivos característicos de la economía capitalista moderna han recibido, desde mediados del siglo pasado, importantes aportes provenientes tanto de la teoría económica como del management estratégico desde la teoría administrativa.

En el primer campo, Edith Penrose (1959, citada en Nelson y Winter, 1982) identificó los vínculos entre el crecimiento de una empresa, su estructura y la naturaleza de su administración. Posteriormente, el análisis histórico de Chandler (1962 y 1977, citados en Nelson y Winter, 1982) respecto de la estrategia en las empresas y su business and technological management, centered in the environment external to the firm and taking large, multidivisional organizations as benchmarks. Unfortunately, this approach has undervalued or ignored the importance of elements such as the flexibility of this type of organization which can become sustainable advantage sources.

This paper presents a method for strategic analysis in the theoretical view of resource-based theory, as presented in Grant (2010), Rangone (1999) an Hall (1992), which can be easily used by owner/managers of micro, small and medium-size enterprises. This can help to better understand this kind of organizations in our field.

Keywords: resource-based theory; strategic behaviour, strategic planning; firm behaviour.

JEL: D21;B52; M19

relación con la administración, explica la manera como las diversas estrategias subjetivas implementadas por las empresas sirven de guía y orientación frente a la realidad económica que, de acuerdo con el autor, no es interpretable mediante el modelo de optimización neoclásica. De otra parte, a finales del siglo XX Nelson y Winter (1982), desde la perspectiva económica evolucionista, introducen el concepto de 'rutina organizacional' como uno de los pilares de la denominada teoría basada en los recursos y las capacidades empresariales (resource-based theory).

Por su parte, en el campo de la administración, abunda la literatura respecto de metodologías, técnicas y herramientas de gestión administrativa y estratégica, específicas e integrales, cuya unidad de análisis es la gran 
empresa multi-divisional que desempeña funciones especializadas articuladas orgánicamente en procura de un mismo objetivo. El interés teórico y práctico del management estratégico y la organización industrial en la perspectiva administrativa convencional se ubican en la interfase entre la estrategia organizacional y el ambiente externo a la empresa, con poco o escaso énfasis en los recursos particulares de las organizaciones.

En contraste, el análisis estratégico en la perspectiva de la teoría basada en los recursos se ocupa fundamentalmente de la correspondencia entre recursos y capacidades internas a la empresa con las oportunidades que surgen en su entorno. De manera que la estrategia resulta de dos factores principales, por un lado, de los recursos y capacidades de la organización y, por el otro, de la ventaja competitiva desarrollada y sostenida por la empresa.

De hecho, de acuerdo con Grant (2010, p. 125), los recursos y capacidades internas a la empresa se perciben como las bases más seguras para la formulación de la estrategia en la medida en que la globalización en curso de la economía ha contribuido con la inestabilidad de los entornos industriales en sus diversos ámbitos geográficos; por su parte, las ventajas competitivas, más que el atractivo de la industria (como se ha enfatizado desde el management estratégico y la organización industrial), son la fuente primaria de beneficios económicos por encima de los normales.

Respecto de las economías más avanzadas, de acuerdo con Rangone (1999), el análisis estratégico en las pequeñas y medianas empresas (pymes) se ha orientado en dos direcciones, en la primera se muestra la presencia o no del análisis estratégico y las prácticas de planificación; la segunda intenta demostrar la efectividad de tales prácticas en términos del impacto positivo en el desempeño de este tipo de organizaciones. En esta última dirección,
Peteraf (1993) aborda el problema teórico de la estrategia; Rangone (1999) hace una propuesta para el análisis estratégico como el principal soporte para el desarrollo de ventajas competitivas sostenibles; y, más recientemente, Ryan (2003) examina el significado de la estrategia y la planeación estratégica en las pymes irlandesas.

En cualquier caso, este tipo de análisis no ha sido explícitamente abordado en relación con las micro, pequeñas y medianas empresas (mipymes) de los países menos desarrollados, en los cuales la discusión se ha orientado primordialmente al problema global de la gestión integral o se ha abordado como parte del problema de la gestión del conocimiento. Las mipymes en las economías menos avanzadas, pese a las enormes dificultades derivadas de sus altos niveles de informalidad, la menor dotación de recursos y la ausencia de economías de escala (Aragón y Sánchez, 2002), tienen reconocida e importante presencia en términos de generación de empleo y del producto; asimismo, a ellas se les reconocen ciertas ventajas competitivas potenciales y reales asociadas con la simplicidad de su organización, la flexibilidad y la rapidez de adaptación.

En este artículo se aborda el problema teórico y metodológico del análisis y la práctica estratégica en mipymes en la perspectiva teórica de los recursos y capacidades empresariales que concibe y explica la competitividad de las organizaciones a partir del grado de especialización de sus activos (recursos) y del desarrollo de competencias (capacidades) en el tiempo. De este modo, el análisis estratégico, el cual subyace a la creación de ventajas competitivas sostenibles y, por lo tanto, a la competitividad, se refiere al empleo eficiente y eficaz de recursos y capacidades.

El objetivo central del trabajo es aportar elementos teóricos y prácticos que sirvan de soporte en la construcción 
de una herramienta de uso práctico que permita traducir la orientación estratégica de la empresa en objetivos mensurables. De manera explícita, se aborda la dimensión estratégica como un factor determinante del posicionamiento competitivo de las mipymes, a partir del reconocimiento de sus recursos y capacidades internas.

Estas empresas, con sus debilidades y fortalezas, son una realidad del mundo en desarrollo que amerita un análisis preciso de sus diversas y complejas dimensiones. El estudio teórico y práctico de la estrategia en este tipo de organizaciones empresariales se adopta al enfoque de la teoría basada en los recursos y capacidades empresariales que pone el acento en "...la importancia de la especificidad y la dificultad de imitación de los recursos que soportan el desempeño"" (Nelson y Winter, 1982, p. 8).

El análisis estratégico y la aproximación metodológica a la práctica estratégica empresarial presentados en este documento tienen fundamento teórico en los artículos de Rangone (1999, 1998, 1997), Grant (2010, 1991) y Ryan (2003), entre otros, quienes proponen el desarrollo de ventajas competitivas dinámicas en empresas pequeñas y medianas en las economías desarrolladas a partir del reconocimiento de la dotación y el alineamiento estratégico de sus recursos y capacidades que las diferencian de sus competidores directos y potenciales. En ellos, las empresas, concebidas como “....un conjunto único de recursos y capacidades heterogéneas, ... [se convierten] en el elemento clave del análisis estratégico" (Carablancas, 2011, p. 14).

El documento contiene tres partes adicionales; en la segunda se presentan, a manera de antecedente, algunos elementos relevantes en el análisis y la práctica estratégica

1 Esta, al igual que todas las citas de artículos y libros publicados originalmente en inglés, corresponde a una traducción del autor. en las pequeñas y medianas empresas (pymes), especialmente los desarrollos de Porter (1991, 1985, 2000) y, como centro del análisis, los elementos teóricos introducidos por la teoría de los recursos y capacidades. En la tercera parte se aborda directamente el problema de la formulación estratégica en pymes fundamentada en recursos y capacidades de la empresa capaces de generar, sostener y permitir la apropiación de los beneficios económicos derivados de las ventajas competitivas. En la cuarta y última parte, a modo de conclusión, se presenta el modelo práctico para el análisis y la formulación estratégica en las mipymes.

\section{Análisis y práctica estratégica en las organizaciones empresariales}

Un plan o estrategia en una organización empresarial formulado con el objetivo de alcanzar y sostener alguna ventaja competitiva implica, necesariamente, "la correspondencia de las actividades del negocio con el entorno y los recursos disponibles" (Ryan, 2003, p. 4). En este sentido, de acuerdo con O'Regan and Ghobadian (2002), la estrategia es un concepto subjetivo, identificable por administradores, accionistas e inversionistas y empleados claves de la empresa, que permite definir un curso de acción a seguir, con implicaciones en el largo plazo.

Una proposición básica en el campo del management estratégico, sostiene que las empresas deben tomar decisiones o elegir cursos de acción para influir en el éxito o fracaso en la competición con otras empresas en los mercados; tales decisiones se refieren fundamentalmente a

...la selección de metas; la elección de productos y servicios a ofrecer; el diseño y la selección de políticas determinantes del posicionamiento en el mercado del producto (esto es, la estrategia competitiva); la elección de un nivel 
apropiado de alcance y diversidad; y el diseño de la estructura organizativa, el sistema administrativo y las políticas en la definición y coordinación del trabajo (Rumelt, Schendel y Teece, 1991, p. 6).

Respecto a la competitividad empresarial y el análisis estratégico, la llamada "revolución administrativa" iniciada después de la segunda guerra mundial puso el énfasis en el conocimiento y la "gestión tecnológica" como principal generador de valor económico (Baena, Botero y Montoya, 2003), especialmente, en los problemas relacionados con su generación, las actividades de Investigación y Desarrollo $(\mathrm{I}+\mathrm{D})$ o investigación básica en procura de su ampliación, asimilación, aplicación y transformación, como principal soporte de la competitividad de las empresas. No obstante, la gestión tecnológica eficiente no es condición suficiente de la competitividad empresarial, especialmente en el caso de las mipymes en el ámbito de las economías de menor desarrollo, dadas sus limitaciones específicas para el acceso, la generación y empleo de conocimiento con fines productivos.

Posteriormente, durante los años ochenta del siglo XX, simultáneamente con los trabajos de organización industrial de Michael Porter (1980, 1985), emerge el nuevo enfoque denominado "teoría basada en los recursos y capacidades" de la empresa que enfatiza en la importancia de la especificidad y la dificultad de imitación de los recursos que soportan su desempeño en el mercado. Desde esta perspectiva teórica, la estrategia trata fundamentalmente de la dirección de las organizaciones (en este caso, empresas) en lo que respecta a la correspondencia entre sus recursos y capacidades con las oportunidades que surgen en el entorno (Grant, 2010, p. 123). De este modo, el énfasis del análisis y la práctica estratégica recae en los recursos de la organización como fuente de capacidades competitivas, y no en la identificación de oportunidades en el entorno externo de la empresa como sucede en los campos de la organización industrial.

\subsection{Análisis y planeación estratégica en pymes}

Además de las restricciones financieras y la limitación de los recursos, los cuales dificultan la innovación y limitan el crecimiento potencial de las pymes, existen algunas dificultades asociadas con la planeación y práctica estratégica en este tipo de organización empresarial. O'Regan y Ghobadian (2002, p. 665) identifican barreras internas y externas para el análisis y la práctica estratégica. Entre las primeras, señalan los problemas de comunicación entre empleados y trabajadores, la duración temporal exigida por la implementación de planes, limitaciones de las capacidades de los empleados, falta de comprensión de las metas de la empresa por parte de empleados y trabajadores y falta de efectividad de la coordinación en la implementación de los planes; por su parte, entre las principales barreras externas se destacan las dificultades introducidas por cambios imprevistos en el ambiente, las crisis que distraen la atención en la implementación y todos los factores externos que puedan afectar la implementación de planes estratégicos.

Adicionalmente, la planificación de las actividades por parte de pymes que operan en ambientes dinámicos requiere de revisiones, modificaciones y ajustes permanentes, los cuales deben ser convenientemente registrados y analizados para introducir nuevos contenidos y cambios en las prácticas, para el logro de objetivos de largo plazo. Pero, más allá del registro y seguimiento de planes y resultados, el entorno cambiante implica para las empresas el carácter emergente de la estrategia, cuya documentación y comunicación a las diferentes partes de la organización involucradas puede ser de enorme dificultad.

Ryan (2003) distingue dos perspectivas del análisis estratégico que se adecúan a las características de las pymes: la organización industrial, especialmente los trabajos de 
Michael Porter y la teoría basada en los recursos. En el primer caso, en el cual el énfasis analítico se encuentra en el entorno o ambiente de la organización, Stonehouse y Pemberton (2002, p. 854) identifican cuatro tipos básicos de estrategias:

1. Prescriptiva (deliberada o planeada), que, propia de las grandes compañías, describe la planeación de largo plazo para alcanzar la correspondencia entre estrategia organizacional y el entorno de las grandes organizaciones.

2. Emergente (aprendizaje), que introduce la posibilidad de los ajustes o cambios de acuerdo con la necesidad.

3. Posicionamiento competitivo (tipo Porter) centrada en el entorno competitivo.

4. Competencial centrales o basada en los recursos, centrada en los recursos y las capacidades de la empresa.

De ellas, los autores escogen la estrategia del tipo emergente como representativa de pymes, en tanto que, frente a las condiciones cambiantes del entorno, permite dar respuestas también variables (flexibles) a partir de la dotación de recursos de la empresa (p. 858, cursiva nuestra), como se explica en los trabajos de Porter sobre competitividad empresarial y, especialmente, respecto de la cadena de valor.

Por su parte, el análisis estratégico en la perspectiva teórica de los recursos y capacidades, puede ser más adecuado para la planeación y la práctica en pymes en tanto que, a diferencia de la primera, implica una revisión global centrada en los recursos y capacidades de la empresa, sin abandonar el contexto. La planeación estratégica, de este modo, permite reconocer, por ejemplo, que los empleados pueden estar en mejor posición para advertir la misión de la organización y para contribuir de manera efectiva con las actividades involucradas en la producción o la provisión de servicios de alto valor para los compradores; asimismo, que pueden aportar en la implementación de los planes estratégicos emergentes de estas organizaciones. No menos importante es que el ejercicio mismo de la planeación estratégica podría contribuir a que el propietario-administrador típico de las pymes supere una de las principales falencias identificadas en los estudios empíricos, el reconocimiento de su importancia, efectos potenciales y consecuencias.

\subsection{La cadena de valor de Michael Porter y la estrategia empresarial}

El modelo de la cadena de valor (Porter, 1985, 1991, 1996) es una interpretación convencional de la práctica estratégica que, inscrita en el paradigma, estructura, conducta, desempeño, se centra en los aspectos operacional y económico de la estrategia empresarial. La cadena de valor de una empresa describe el conjunto de actividades primarias (proceso de producción) y de soporte (provisión de los insumos adquiridos) que se relacionan y compiten entre sí con el propósito de contribuir a la generación de valor para el comprador (Ryan, 2003).

La cadena de valor refleja el funcionamiento de la empresa como un sistema compuesto orgánicamente de actividades diversas; como herramienta de uso práctico permite la identificación de la(s) actividad(es) que tienen significado estratégico para la empresa. De acuerdo con Ryan (2003), el alineamiento estratégico ${ }^{2}$ (estrategic fit) y el

2 Grado en el cual una organización logra la correspondencia entre sus recursos y capacidades con las oportunidades que brinda el ambiente externo. 
refuerzo de estas actividades son el fundamento del desempeño exitoso y la competitividad.

En síntesis, el modelo de la cadena de valor de Porter "desagrega la empresa en sus actividades estratégicamente relevantes con el fin de comprender el comportamiento de los costos y las fuentes de diferenciación existentes y potenciales" (Porter, 1985, p. 26). Una estrategia genérica ${ }^{3}$ de una empresa, bien sea de liderazgo en costo, diferenciación o enfoque, de acuerdo con Porter (1991, 1985), se compone de las acciones ofensivas o defensivas orientadas a crear una posición defendible dentro de una industria como respuesta al grado de competencia de su entorno por parte de proveedores, compradores, productos sustitutivos, competidores actuales y potenciales (Ayala Ruiz, 2011); su resultado en caso de ser correcta es la obtención de beneficios por encima del promedio en el largo plazo. En relación con las pymes, Porter (1985, p. 264) sostiene que sus objetivos deben concentrarse en servir un nicho de marcado correctamente identificado con bienes de mejor calidad que las alternativas ofrecidas (estrategia de diferenciación), cuyo tamaño sea suficientemente grande como para asegurar la supervivencia de la empresa.

Ryan (2003) atribuye un valor estratégico específico a la cadena de valor, en tanto que es una herramienta de diagnóstico de ventajas competitivas y de identificación de los mecanismos para fortalecerlas. En efecto, en el modelo de la cadena de valor se incluyen los factores determinantes de la competencia que hacen atractivo un sector industrial, así como los factores que cambian con el tiempo y pueden ser modificados mediante la estrategia (Benzaquen, J. et ál., 2010); como resultado de su

3 En Porter (1991) se definen diversas estrategias genéricas elegibles por la empresa, caracterizadas sustancialmente por el alcance (amplio o estrecho) de sus decisiones y por los medios (bajo costo o diferenciación del producto) para alcanzar la ventaja. aplicación en el análisis y la práctica, es posible entender las tendencias en las industrias y la competencia y formular una estrategia global de desarrollo competitivo.

\subsection{La teoría basada en los recursos, la ventaja competitiva y la estrategia en pymes}

La competitividad ${ }^{4}$ depende, además de factores externos a la empresa, de las decisiones gerenciales y de planificación estratégica determinantes de la eficiencia y eficacia de la organización; por ello, involucra tanto elementos de política económica del país o región que afectan el desempeño, como de variables gerenciales y tecnológicas. Adicionalmente, el reconocimiento y desarrollo de las ventajas competitivas en las organizaciones empresariales debe conducir a mejorar el desempeño en el mercado en términos de mayor participación y mejor posicionamiento.

De acuerdo con Grant (2010), de las dos fuentes de beneficios positivos identificadas en la literatura, los atractivos de la industria y las ventajas competitivas, la segunda es la más relevante ${ }^{5}$. La obtención de beneficios económicos por encima del promedio se explica mejor por la presencia de ventajas competitivas desarrolladas y mantenidas en el tiempo a partir de los recursos y capacidades internos a las organizaciones empresariales. Es por ello que

4 Para los propósitos de este trabajo se define competitividad como la "habilidad de las unidades económicas para crear estructuras que permitan una modificación de las condiciones técnicas de la producción (producción basada en conocimiento) y esto lleve al incremento del nivel de vida de los participantes" (López Ortega, s.f.).

5 La distinción entre atractivo de la industria y ventajas competitivas basadas en la superioridad de los recursos de la empresa se fundamenta, como se ha explicado, en la distinción económica de las rentas (ricardianas o monopólicas). 
en las condiciones modernas de internacionalización y liberalización económicas, que han incrementado las presiones competitivas a todo nivel, pocas industrias ofrecen un refugio confortable a una fuerte competición y pocos son los factores de la industria, externos a la empresa, que explican los diferenciales en los beneficios.

A diferencia de la formulación convencional de la estrategia $^{6}$, centrada en la selección de la industria y el posicionamiento, el análisis estratégico en la perspectiva de los recursos y capacidades empresariales pone el énfasis en la especificidad de cada empresa: la clave está en explotar las diferencias y no hacer lo mismo que los otros. En este sentido, desarrollar ventajas competitivas implica formular e implementar una estrategia que explote la especificidad de su dotación de recursos y capacidades (Grant, 2010, p. 128).

El énfasis en los recursos y capacidades internos a la organización no implica, sin embargo, el desconocimiento de los factores claves de éxito en la industria ${ }^{7}$, externos a las empresas, que también son determinantes en la obtención de beneficios económicos. Por ello, la administración de la empresa al adoptar una estrategia coherente puede influir en el desarrollo y disposición de los activos, recursos y capacidades estratégicos (Amit y Schoemaker, 1993).

En síntesis, la formulación de la estrategia en este enfoque se fundamenta en el conocimiento y la comprensión completa y profunda de los recursos, desempeños y competencias de la empresa. Esto permite, en primer lugar, la selección de la estrategia que explote las fortalezas claves de la empresa; y, segundo, por su énfasis constructivo más

6 Contenida en los estudios de organización industrial y el management estratégico.

7 Que en la perspectiva del diamante competitivo de Porter corresponden a los 'factores claves de la industria'. que descriptivo, crear y desarrollar recursos y capacidades de la empresa que soporten la ventaja competitiva hacia el futuro. Al respecto, Peteraf (1993) identifica cuatro condiciones teóricas que, interrelacionadas, subyacen al desarrollo de las ventajas competitivas empresariales en la perspectiva de la teoría basada en los recursos: la heterogeneidad de los recursos, los límites ex post a la competición, la movilidad imperfecta de los factores y los límites ex ante a la competición.

\subsubsection{Las características de los recursos empresariales}

Como condiciones o características fundamentales para el desarrollo de ventajas competitivas, la heterogeneidad de los recursos hace posible la creación de rentas ricardianas o monopólicas; los límites ex post evitan la competencia por las rentas y permiten sostenerlas en el tiempo; la movilidad imperfecta asociada a la heterogeneidad de los recursos asegura que los recursos valiosos permanecen en la empresa y que las rentas pueden ser compartidas con los dueños de tales recursos; y, finalmente, los límites $e x$ ante a la competición hace costoso entrar a competir por las posiciones ventajosas.

La heterogeneidad de los recursos. Dada la existencia de niveles diferenciados de eficiencia de los recursos productivos, las empresas se encuentran en condiciones de producir a costos diferenciados y de atender las necesidades de los clientes de manera también diferenciada; aquellas dotadas de recursos de eficiencia superior y mejores capacidades estarán en condiciones de apropiar rentas. Sobre este particular, en la literatura se señala la consistencia entre la heterogeneidad de los recursos con los modelos de obtención de rentas ricardiana y rentas monopólicas derivadas del poder de mercado. 
Cabe precisar que, de acuerdo con la teoría ricardiana, la heterogeneidad de los recursos se explica por la presencia de factores productivos de eficiencia superior cuya oferta es limitada en una industria; por ello, la empresa o empresas que los poseen pueden producir a costos medios inferiores, percibiendo beneficios por encima de lo normal en la forma de rentas pagadas a ellos. Estos no son atribuibles a ningún poder de mercado o a restricciones impuestas a la competición; asimismo, podrán ser sostenibles en el tiempo solamente si la oferta de los recursos más eficientes no se expande y si no son imitados (Peteraf, 1993).

En contraste, los beneficios monopólicos provienen de una restricción deliberada de la oferta del producto; por ejemplo, en el modelo del monopolio la heterogeneidad puede resultar de la diferenciación del producto o la competición espacial (Peteraf, 2003, p. 182) debido a la presencia de barreras a la movilidad intraindustrial que termina por diferenciar las empresas, entre otras características de una industria. En todo caso, las empresas con poder de mercado, enfrentadas a curvas de demanda con pendiente negativa, estarán en condiciones de obtener beneficios positivos al restringir la oferta de producto. Teóricamente, firmas homogéneas podrían obtener rentas monopólicas, dependiendo de la presencia de pocos competidores en el mercado y, asociado a ello, de la capacidad de imponer barreras a la entrada de nuevos competidores.

Los limites a la competición 'ex post'. Son las fuerzas que limitan la competencia una vez que la empresa está en capacidad de obtener rentas; estos límites garantizan, como principio estratégico, la permanencia en el tiempo de la heterogeneidad que da origen a las rentas, sean estas ricardianas o monopólicas. Estos límites son necesarios en tanto que la competición ex post al logro de una posición ventajosa en la industria puede disipar la renta ricardiana al hacer la curva de oferta más elástica; del mismo modo, al incrementarse la oferta de producto o al ser más elásticas las curvas de demanda individual, se erosionan las rentas monopólicas (Peteraf, 2003, p. 182).

Los dos factores críticos que limitan la competición ex post son la imitabilidad y la sustitubilidad imperfectas. Esta reduce las rentas al hacer más elásticas las demandas que enfrenta el monopolista u ologopolistas; la imitabilidad imperfecta garantizada por los denominados 'mecanismos de aislamiento' (Rumelt, 1984; citado en Peteraf, 2003) protegen a la empresa de la imitación y contribuyen a preservar las rentas; entre ellos se encuentran los derechos de propiedad sobre los recursos escasos y varios cuasi-derechos derivados de los rezagos, asimetrías en la información y fricciones que impiden la imitación.

Otros mecanismos cuando se emplean activos especializados son el aprendizaje por parte de los productores, la reputación y las economías de escala. Peteraf (2003) relaciona la imitabilidad y la sustitubilidad con las características del proceso de acumulación de activos; estos, especialmente si son no transables, en lugar de ser adquiridos en los mercados, se acumulan en el tiempo.

La movilidad imperfecta de los recursos. Los recursos se consideran perfectamente inmóviles si no pueden ser objeto de comercio, como en el caso de aquellos cuyos derechos de propiedad no se encuentran bien definidos o que son idiosincráticos a la empresa, por lo cual no pueden ser empleados por fuera de ella. En un nivel menos restrictivo, los recursos de movilidad imperfecta, a pesar de poder ser objetos de comercio, tienen más valor en la empresa que los poseen y emplean en comparación con cualquier otro uso; son recursos especializados a las necesidades específicas de las empresas. También son recursos con movilidad imperfecta aquellos co-especializados, cuyo uso es complementario con algún otro o que tiene un alto valor económico cuando se emplean juntos; o aquellos cuyos 
costos de transacción asociados a su transferencia son excesivamente altos (Williamson, 1975).

Los recursos de movilidad imperfecta, por su naturaleza, pueden permanecer en la empresa aun en el largo plazo y convertirse en fuente de ventajas sostenibles. De acuerdo con Peteraf (2003), la diferencia entre el valor de un recurso de movilidad imperfecta para una empresa y su costo de oportunidad $^{8}$ es una forma de renta, también llamada cuasi-renta: el exceso del valor de un activo sobre su valor de salvamento o su valor en el próximo mejor uso. De este modo, en la ausencia de rentas ricardianas o monopólicas, un activo puede generar 'cuasi-rentas apropiables' en razón del carácter imperfecto de su movilidad (p. 184).

En suma, las ventajas competitivas sostenibles asociadas a la movilidad imperfecta surgen del hecho de que los recursos pueden permanecer disponibles para la empresa, lo cual los hace difícil de imitar; o, en caso de que este tipo de recursos no pertenezcan a la empresa, de la posibilidad de compartir las rentas entre la empresa y el dueño del recurso, bien porque la primera está obligada al uso de un recurso especializado o porque las rentas puedan ser producidas y apropiadas conjuntamente.

Los limites 'ex ante' a la competición. De acuerdo con esta característica, antes de que una empresa establezca una posición superior de un recurso, debe existir competición limitada por dicha posición en la industria; esto es, una posición superior es fuente de beneficios adicionales si alguna empresa tiene la visión o la buena fortuna de adquirir el recurso en ausencia de competición. En caso de que esta última exista, es más probable que dichos beneficios se reparten entre las empresas participantes. Por ello, el

8 El costo de oportunidad aquí, diferente a su concepción convencional, se refiere al valor del recurso para el segundo usuario potencial que más valora el recurso. desempeño económico depende no solo de los ingresos derivados de las estrategias, sino también de los costos de implementación de la estrategia; los beneficios por encima de lo normal se derivan de la incertidumbre $e x$ ante que hacen posible el posicionamiento de un recurso (Peteraf, 2003, p. 185).

\subsubsection{Los recursos y capacidades de la empresa}

La relación entre recursos y capacidades de la empresa se fundamenta en la idea de que "There is a close relation between the various kinds of resources with which a firm works and the development of ideas, experience, and knowledge of its managers and entrepreneurs" (Penrose, 1959, citada en Rangone, 1999), la cual, en la perspectiva de la formulación de la estrategia empresarial concede primordial importancia a las características internas de la organización. La estrategia en la perspectiva teórica basada en los recursos se ocupa de la correspondencia entre recursos y capacidades de la empresa con las oportunidades que surgen en su entorno (Grant, 2010, p. 125). Los recursos son los activos productivos de la empresa, insumos empleados en el proceso productivo que individualmente no confieren ventajas competitivas, pero que puestos en acción de manera conjunta pueden crear capacidades organizacionales. En general, de acuerdo con Rangone (1999), Grant (2010, 1991) y Hall (1992) existen tres categorías básicas de recursos empresariales: tangibles, intangibles y humanos ${ }^{9}$.

Recursos tangibles. Son los activos físicos y recursos financieros, estos tienen un valor contable que no necesariamente representa el valor del recurso para la empresa.

9 Rangone (1999) incluye los recursos humanos en el grupo de los recursos tangibles. Por su parte, Grant (1991) en una clasificación compatible identifica seis categorías de recursos: físicos, financieros, tecnológicos, reputación, organizacionales y humanos. 
- Activos físicos: Equipamiento, planta, bienes inmuebles, etc.

- Recursos financieros: monetarios, acciones, capacidad de endeudamiento, entre otros.

Recursos intangibles. Recursos cuyo valor reside en la confianza del consumidor, expresado en el mayor precio que están dispuestos a pagar, relativo a los otros compradores en el mercado (Grant, 2010).

- Conocimiento tecnológico: patentes, derechos de propiedad, secretos industriales y comerciales.

- Reputación (reputacional assets): marca (brands), recursos relacionales.

- Cultura organizacional.

Recursos humanos. Son el conocimiento, la experiencia y el esfuerzo que tienen y ofrecen los empleados a la empresa.

- Habilidades y saber hacer (know-how)

- Capacidades de comunicación y colaboración

- Motivación

Por su parte, Prahalad y Hamel (1990) definen las capacidades como las verdaderas fuentes de nuevos productos y el fundamento de la estrategia en los ambientes competitivos. De manera específica, son las habilidades de un grupo de recursos para, conjunta y armoniosamente, desempeñar una tarea o actividad (Grant, 1991). En la literatura especializada, de acuerdo con Rangone (1999), se identifican tres capacidades empresariales básicas: producción, innovación y mercadeo.
Innovación. Habilidad para desarrollar nuevos productos y procesos y lograr desempeños tecnológicos y administrativos superiores.

Producción. Habilidad de producir y entregar los productos a los consumidores al mismo tiempo que se asegura la superioridad competitiva (calidad, flexibilidad, costo, entre otros).

Mercadeo (marketing). Habilidad de la empresa para vender sus productos de manera efectiva y eficiente.

Finalmente, Grant (2010) precisa el vínculo entre recursos, capacidades y ventajas competitivas. Al funcionar conjuntamente los recursos pueden crear capacidades organizacionales, la esencia del desempeño superior de la empresa. En la figura 1 se sintetiza, de acuerdo con lo previamente expuesto, la relación entre estas categorías y su implicación para el análisis estratégico.

Figura 1. Relación entre recursos, capacidades y ventaja competitiva

VENTAJA COMPETITIVA SOSTENIBLE

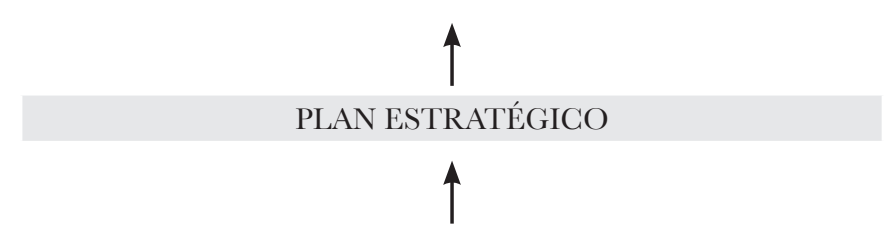

CAPACIDADAES: producción, innovación y mercadeo

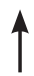

RECURSOS: tangibles, intangibles y humanos

Fuente: elaboración propia a partir de Grant (2010, p. 131). 


\section{Recursos, capacidades y estrategia empresarial}

Desde los años noventa del siglo XX se han hecho importantes contribuciones a la construcción de un marco analítico de la estrategia empresarial en la perspectiva de la teoría basada en los recursos (Peteraf, 1993; Amit y Schoemaker, 1993; Rangone, 1999), junto a algunos esfuerzos orientados a estudios prácticos de las implicaciones de la teoría con respecto a la formulación de estrategias (Grant, 2010; Ryan, 2003).

En este apartado se presentan los elementos del análisis y la práctica estratégica cuyo eje articulador son los recursos y capacidades de las pymes. Con respecto a las mipymes de las economías de menor desarrollo la característica más sobresaliente del análisis radica en que la unidad de referencia no es la gran empresa multi-divisional, sino los recursos pertenecientes a ellas y las capacidades o competencias efectivas para la producción, el mercadeo y/o la innovación.

\subsection{Un nuevo enfoque: la estrategia fundamentada en recursos y capacidades empresariales}

Los recursos y capacidades estratégicos de la organización definen lo que la empresa es capaz de hacer; estratégicamente proporcionan la dirección básica a seguir y son las fuentes primarias de beneficios para la empresa derivados de rentas ricardianas y/o monopólicas. En consecuencia, se trata del "...diseño de una estrategia que haga el uso más efectivo de los recursos y capacidades centrales" (Grant, 1991, p. 129); la esencia del análisis y la práctica estratégica con este enfoque teórico radica en la disyuntiva que surge entre desarrollar activos especializados para incrementar los beneficios y la reducción de la flexibilidad al enfrentar la competencia schumperiana por rentas. La ventaja competitiva se genera y se sostiene cuando los competidores existentes y potenciales carecen de la habilidad o el deseo de imitar (o adquirir) los recursos y capacidades que generan rentas para la empresa que los posee y controla (Amit y Schoemaker, 1993, p. 40).

El análisis y la planeación estratégica parte del reconocimiento de las condiciones particulares de escasez, complementariedad, movilidad limitada y las condiciones de apropiabilidad de los recursos y capacidades que permiten la obtención de rentas por parte de las empresas. Asimismo, permite dar cuenta de la incertidumbre y complejidad propias del ambiente externo a la empresa (industria), así como de las imperfecciones en los mercados de factores que contribuyen a ahondar las diferencias entre las empresas.

Más que la búsqueda de rentas monopólicas característica de la formulación estratégica en la perspectiva de la organización industrial, el elemento esencial de la formulación estratégica desde este enfoque consiste en la obtención de rentas ricardianas derivadas de aquellos recursos y capacidades únicos y difíciles de imitar. Los recursos y capacidades de las empresas constituyen la base segura sobre la cual se define la identidad de la organización empresarial, en cualquier caso más estable que la proporcionada por las condiciones cambiantes del entorno (industria).

Existen dos características de la formulación estratégica desde esta perspectiva: el carácter limitado del alcance de la estrategia y el tiempo. En el primer caso, el alcance estratégico de la empresa se limita a aquellas actividades o funciones en las cuales la ventaja competitiva sea clara y definida. 
En relación con el tiempo, la habilidad de los recursos y capacidades de una empresa para sostener una ventaja competitiva es esencial en la definición del horizonte de la planeación estratégica. Por ello, si los recursos y capacidades son de baja durabilidad o fácilmente transferidos o replicados, como sucede en las primeras fases de la introducción de una nueva tecnología, entonces conviene adoptar una estrategia de agotamiento de corto plazo o invertir en el desarrollo de nuevas fuentes de ventajas competitivas; este tipo de recursos solo pueden sostener ventajas competitivas en mercados pequeños poco atractivos o si es posible ocultar la existencia de las ventajas competitivas (Grant, 1991, p. 133). Por su parte, en caso de que las ventajas competitivas se fundamenten en la diferenciación e innovación fácilmente imitable, las empresas cuentan con una ventana de oportunidades estrecha para explotarlas; deben preocuparse no por sostener la ventaja existente, sino por generar flexibilidad para generar ventajas a mayor velocidad que la de la imitación.

\subsection{La formulación de la estrategia en mipymes}

El análisis estratégico en pymes involucra tanto las especificidades de las organizaciones como las de sus entornos en constante cambio (la industria, la economía nacional o mundial, entre otros) y se resuelve, de acuerdo con Kantis (1996), en la realización de un conjunto de acciones más o menos complejas, en una secuencia temporal determinada, en procura del fortalecimiento competitivo. Así, la exploración estratégica de la capacidad para generar respuestas innovadoras y de la flexibilidad de las mipymes contribuye con el desarrollo de ventajas competitivas sostenibles.

\subsubsection{Elementos de la estrategia}

La competitividad de largo plazo de una empresa depende de su dotación de recursos que la hacen diferente de sus competidores en razón de la dificultad para imitarlos, durabilidad y posibilidades de sustitución. Según Prahalad y Hammel (1990), los principales elementos de la estrategia empresarial son: el alcance estratégico de los recursos, las capacidades, las ventajas competitivas y la formulación de la estrategia.

Los recursos estratégicos de la empresa. Grant (2010) clasifica los innumerables recursos con los que cuenta la empresa, de acuerdo con su carácter, en recursos tangibles, intangibles y humanos; en la tabla 1 se presenta el esquema básico para la clasificación de recursos de las empresas.

De la enorme diversidad de recursos de las organizaciones empresariales, existen los denominados 'recursos críticos' o 'estratégicos' en tanto que son la base material o inmaterial de la ventaja competitiva sostenible de la empresa. Rangone (1999) define el carácter crítico o estratégico de los recursos mediante ensayos (tests) de las características que soportan la ventaja competitiva, entre estas:

- Imitabilidad: medida de la dificultad de los competidores reales y potenciales para imitar el recurso debido a la especificidad física, trayectoria de desarrollo o impedimento económico.

- Duración: medida de los beneficios que el recurso generará en el largo plazo.

- Sustitubilidad: medida de la dificultad de los competidores para remplazar un recurso con una alternativa que le proporcione la misma ventaja. 
- Superioridad competitiva: medida en la que el recurso contribuye con diferencias a las empresas competidoras.

- Apropiabilidad: verificación de la capacidad de la empresa para explotar la ventaja generada en el mercado.

Tabla 1. Recursos genéricos de la empresa

\begin{tabular}{|c|c|}
\hline CLASES & SUBGLASES (ejemplos) \\
\hline Tangibles & $\begin{array}{l}\text { - Físicos: planta, maquinaria, equipos } \\
\text { - Financieros. }\end{array}$ \\
\hline Intangibles & $\begin{array}{l}\text { - Activos: legales (contratos, licencias, patentes) y } \\
\text { no legales (reputación, bases de datos) } \\
\text { - Competencias: "know how" (administradores, tra- } \\
\text { bajadores y accionistas) y cultura organizacional } \\
\text { (capacidad de aprendizaje). } \\
\text { - Tecnología: patentes, derechos de propiedad, se- } \\
\text { cretos industriales y comerciales. } \\
\text { - Reputación: marcas, relaciones } \\
\text { - Elementos de la cultura empresarial }\end{array}$ \\
\hline Humanos & $\begin{array}{l}\text { - Habilidades: lo que la empresa es capaz de hacer } \\
\text { - Capacidades: comunicación, colaboración. } \\
\text { - Motivación }\end{array}$ \\
\hline
\end{tabular}

Fuente: elaboración propia a partir de Grant (2010) y Rangone (1999).

Las capacidades organizacionales (empresariales). Se definen como las habilidades (competencias) de las empresas para disponer sus recursos para el desempeño de una tarea o actividad con el propósito de alcanzar un resultado final (Helfat y Lieberman, 2002). De especial importancia son las competencias de la empresa que le permiten hacer bien las cosas en comparación con sus competidores (Grant, 2010), también denominadas 'núcleo de competencias' en Prahalad y Hammel (1990), esenciales para su desempeño y estrategia. Estas presentan como características distintivas dos hechos:
- Contribuyen de manera significativa al valor proporcionado al cliente o la eficiencia con la cual se provee dicho valor, y

- ofrecen una base para entrar en nuevos mercados (Grant, 2010, p. 135).

Para la identificación de las capacidades estratégicas de la empresa se hace necesario desagregar y clasificar las actividades ${ }^{10}$ mediante el análisis funcional o de la cadena de valor (Grant, 2010). En el primer caso, las capacidades organizacionales se relacionan con las áreas funcionales de la empresa, entre las cuales se incluyen: los aspectos operacionales, diseño de productos, mercadeo, manejo de la información, investigación y desarrollo, entre otras. El análisis de la cadena de valor, por su parte, separa las actividades genéricas de la empresa en actividades primarias, aquellas implicadas en la transformación de los insumos y de interfase con el cliente (logística externa, operaciones, logística interna, mercadeo y ventas y servicio al cliente), de las actividades de soporte de la organización (infraestructura, administración del recurso humano, desarrollo tecnológico y aprovisionamiento).

Respecto a las pymes, Rangone (1999) señala la relevancia de tres capacidades básicas: producción, innovación y mercadeo. Estas constituyen el núcleo (core) de la estrategia, bien sea que se presenten de manera individual o en conjunto. Se debe mencionar que Rangone no incluye la financiación dentro de este grupo, porque considera que este ámbito de la organización empresarial se define sustancialmente en el ámbito del mercado (entorno) y, desde el punto de vista interno a la organización, queda incluido en sus desempeños claves en respuesta, por ejemplo, a la escasez de los recursos. 
Cualquiera sea el método de análisis de las capacidades de la organización, funcional o de la cadena de valor, las capacidades existen en un orden jerárquico que incluye desde las amplias y genéricas hasta las capacidades especializadas, desempeñadas por pequeños grupos de recursos de la empresa, que conforman las primeras. En general, las capacidades requieren del trabajo en equipo de varios individuos, con diversas experticias integradas al capital (maquinaria y equipo), tecnología y otros recursos. Nelson y Winter (1982), citados en Grant (2010, p. 137), emplean el término 'rutina organizacional' para referirse a los "patrones de actividades regulares y predecibles compuestas de secuencias de acciones coordinadas de los individuos"; rutinas que se pueden desarrollar a través del aprendizaje en la práctica y que son los bloques constructivos de las capacidades de la empresa.

Las ventajas competitivas. Los recursos estratégicos y las capacidades críticas soportan las destrezas que la empresa puede explotar en el mercado; asimismo, proporcionan el foco de su estrategia de largo plazo centrada en la obtención de los beneficios en el largo plazo. De acuerdo con Grant (2010), la ganancia de beneficios económicos mediante la explotación de recursos y capacidades depende de tres factores: la habilidad para establecer la ventaja competitiva, para sostenerla y apropiar los beneficios derivada de ella; habilidades que, a su vez, dependen de las características de sus recursos y capacidades (p. 137).

De acuerdo con este autor, para establecer o generar ventajas competitivas se requieren dos condiciones mínimas en los recursos y las capacidades de la empresa: escasez y relevancia.

- Escasez: en principio, un recurso o capacidad de amplia disponibilidad en la industria, aunque esencial para competir, no confiere ventaja competitiva alguna.
- Relevancia: un recurso o capacidad debe ser relevante respecto de los factores claves de éxito en el mercado, lo cual le permite contribuir efectivamente en la generación de ventajas para la empresa.

Luego de establecida, la ventaja puede ser sostenible en el tiempo si los recursos y capacidades de la empresa son durables y difícilmente imitables por los rivales, esto es, si no pueden ser transferibles o replicables. En síntesis, la sostenibilidad de la ventaja competitiva está determinada por el grado de durabilidad, movilidad y replicabilidad de recursos y capacidades:

- Durabilidad. Determinada por la velocidad del cambio tecnológico en la industria; una mayor velocidad acorta la vida útil de recursos y capacidades, incluyendo el equipo de capital y las propiedades tecnológicas.

- Movilidad. Definida como la movilidad de recursos y capacidades entre empresas mediante compra y venta; las dificultades para transferir los recursos o la pérdida de valor asociada a ella contribuyen a sostener las ventas competitivas en el tiempo. Las principales fuentes de inmovilidad son geográficas (en el caso de los recursos naturales), el tamaño del equipo de la maquinaria y el equipo, las limitaciones a la movilidad del recurso humano, las asimetrías de información respecto de la calidad de los factores físicos y humanos, las complementariedades de los recursos $y$, en general, las capacidades organizacionales que dependen de conjuntos de factores, entre otros.

- Replicabilidad. Propiedad definida por la complejidad y flexibilidad de las rutinas organizacionales implicadas en la capacidad de la organización. Aún cuando la replicación sea posible, las empresas poseedoras de los recursos y capacidades acumuladas 
en el tiempo solo podrán ser replicadas a altos costos para los posibles rivales.

En última instancia, la empresa dueña de recursos estratégicos y capacidades críticas está en condiciones de apropiar beneficios económicos por encima de los normales en una industria. No obstante, en los casos en los que las capacidades reposan en el recurso humano, cuya característica esencial es que no es propiedad de la empresa como los recursos físicos, la apropiación de los beneficios no es del todo expedita y puede estar mediada por arreglos laborales e incentivos para garantizar la permanencia. En general, en la medida de la menor claridad en la definición de los derechos de propiedad con respecto a recursos y capacidades, mayor es la importancia del poder de negociación relativo en la determinación de la repartición de las rentas.

\subsubsection{Tipos genéricos de estrategias}

Con fines analíticos Miles y Snow (1978), citados en Aragón y Sánchez (2003), establecen una tipología de estrategias a partir del concepto "...de la organización como un sistema complejo e integrado en interacción dinámica con su entorno" y en atención a las siguientes premisas fundamentales:

1) las empresas de éxito desarrollan un enfoque sistemático e identificable de adaptación a su medio ambiente, es decir, dan respuesta al ciclo adoptando un modelo de comportamiento determinado;

2) en cada sector se pueden identificar cuatro orientaciones estratégicas, de modo que las empresas se clasifican en defensivas, exploradoras, analizadoras y reactivas; $y$,

3) cualquier orientación - defensiva, exploradora o analizadora- puede conducir a un buen resultado empresarial (p. 71).
La orientación estratégica defensiva es seguida por aquellas empresas concentradas en el ámbito del producto mercado; es decir, empresas expertas en un área determinada que no buscan nuevas oportunidades fuera de su negocio, sino que tratan de proteger su cuota de mercado y, por ello, requieren de pocos ajustes en tecnología, estructura organizativa o en sus procesos. Orientadas estratégicamente hacia su interior enfatizan en la reducción de costes y el incremento de su eficiencia; sus estructuras organizativas tienden a ser de tipo funcional, es decir, centralizadas y con líneas de autoridad bien definidas.

Con un patrón estratégico explorador, conceptualmente opuesto al defensivo, la empresa busca permanentemente nuevas oportunidades de mercado mediante procesos de innovación y desarrollo de productos que suelen propiciar cambios en el sector; asimismo, procura actuar rápidamente ante las tendencias emergentes del entorno. En general, las empresas cuentan con una estructura organizativa divisional o por productos, son descentralizadas y flexibles en su estilo de dirección.

Las organizaciones cuya orientación estratégica es analizadora, son una simbiosis de las dos anteriores, en tanto que actúan de modo defensivo o explorador en función de las características del entorno y en procura de un equilibrio eficiencia - innovación. En ambientes estables, las organizaciones operan de manera rutinaria, formal y eficiente a través de estructuras y procesos formalizados; sin embargo, en ambientes turbulentos se comportan como seguidores de los competidores más innovadores, tratando de adoptar rápidamente las ideas que parezcan más prometedoras.

Por último, las organizaciones reactivas se caracterizan por la permanente inestabilidad e inconsistencia debido a su incapacidad para responder a los cambios del ambiente, dada la ausencia de la idea estratégica; esta falta 
de consistencia implícita en la estrategia reactiva conduce a los peores desempeños relativos a las tres orientaciones restantes previamente presentadas.

Excepto el comportamiento reactivo, el cual parece una contradicción en los términos, cualquier tipo de estrategia adoptada por la empresa implica un acto consciente cuyo propósito es el posicionamiento competitivo. El comportamiento estratégico se configura y toma forma en la empresa, bien como resultado de un proceso de planificación o de manera emergente, a partir de la definición de un foco estratégico (Mintzberg y Waters, 1985). En todo caso, el proceso de formulación implica el uso de un método o procedimiento que requieren de habilidades y el manejo de herramientas más o menos complejas.

\section{Modelo para el análisis y la práctica estratégica en mipymes}

En este apartado se presenta un modelo guía de análisis estratégico empresarial a partir del reconocimiento e identificación de recursos y capacidades críticas de la organización. Este tipo de modelo concebido originalmente para las pymes en economías desarrolladas (Grant, 2010, 1991; Rangone, 1999, 1998, 1997) se propone para el análisis y la práctica estratégica en mipymes en el contexto de una economía no desarrollada; organizaciones empresariales que, guardadas las proporciones, presentan características asimilables a las pymes y, en cualquier caso, diferentes de las grandes corporaciones multi-divisionales que han sido el objeto de estudio del análisis estratégico convencional (management estratégico y organización industrial).

El modelo tiene como característica esencial la simplicidad y bajos requerimientos de habilidades especiales en el análisis estratégico, o de sistemas sofisticados de información por parte del cuerpo directivo de la empresa. De manera sustancial, permite al equipo directivo o al propietario administrador típico de las mipymes la conexión con las variables claves que soportan la ventaja competitiva de largo plazo. Adicionalmente, parte del reconocimiento de que las mipymes, de manera implícita o explícita, consciente o inconscientemente, definen un foco estratégico soportado en una o más sus recursos y capacidades básicas.

Como ha quedado establecido, de la diversidad de recursos de la empresa son relevantes los denominados críticos o estratégicos en tanto que, por sus características, se vinculan de manera individual o grupal a las actividades o desempeños claves que permiten generar, sostener y explotar ventajas competitivas. Por su parte, las capacidades críticas relevantes para las mipymes se reducen a la producción, innovación y mercadeo, cuyo desarrollo dependen de la dotación de recursos y del foco estratégico de la empresa.

Los pasos metodológicos contemplados en el análisis estratégico en el modelo son: primero, definición del propósito estratégico y, en relación con ellos, los desempeños claves; segundo, identificación de los recursos estratégicos asociados con los desempeños claves; tercero, examen del valor estratégico de los recursos; cuarto, evaluación de la consistencia estratégica de los recursos y, quinto, definición de opciones estratégicas de la empresa.

\subsection{Propósito estratégico y desempeños claves}

El propósito estratégico de la empresa se define desde su interior por parte del equipo administrativo y de manejo, posiblemente conformado por el propietario/adminstrador de la organización y sus empleados de confianza y 
manejo. En todo caso, esto implica dos niveles de elección vinculados entre sí: la definición de capacidades básicas y de los desempeños claves que las vinculan con los recursos estratégicos.

\subsubsection{Definición de las competencias o capacidades básicas}

Las capacidades o competencias básicas, definidas y fundamentadas en la dotación de recursos de la empresa, sirven de soporte a su ventaja competitiva. En este modelo se consideran la producción, innovación y mercadeo como las capacidades básicas relevantes para las mipymes.

Estratégicamente se sugiere que la empresa oriente sus esfuerzos en una de ellas o, en casos excepcionales, trate de abarcar alguna combinación de las tres capacidades de acuerdo con las exigencias de la industria (competidores) y de los demandantes. La medida en que una empresa hace su elección depende de su foco estratégico, que puede ser definido de manera emergente, como suele suceder en las mipymes (Mintzberg y Waters, 1985) o deliberadamente, a la manera de las grandes empresas multi-divisionales.

\subsubsection{Identificación de los desempeños claves}

Los desempeños claves establecen el vínculo de la empresa con los factores de éxito de la industria y el núcleo de beneficios que la organización desea brindar a sus clientes; implican el empleo de recursos y capacidades para el mejor desempeño respecto de los factores de éxito relevantes en la industria. En función de la capacidad con la que se relacionen, los desempeños claves se dividen en tres categorías: desempeños en la producción (manufactura, en el caso de mipymes industriales), desempeño en el desarrollo de nuevos productos (o servicios) y desempeños en el mercadeo.

Mediante el análisis funcional o de cadena de valor se identifican las secuencias de actividades o desempeños claves ligadas a las competencias de la empresa. Para el caso de mipymes, en el presente modelo, como parte del análisis funcional se asocian desempeños claves a las competencias básicas (producción, innovación, mercadeo) ${ }^{11}$. Como se presenta en la tabla 2, a cada capacidad o competencia se asociará un determinado grupo de desempeños claves.

Tabla 2. Desempeños claves y competencias de la empresa

\begin{tabular}{|c|l|}
$\begin{array}{c}\text { GOMPETENGIAS } \\
\text { (GAPACIDADAES BÁSICAS) }\end{array}$ & \multicolumn{1}{|c|}{ DESEMPEÑOS GLAVES } \\
\hline Producción & $\begin{array}{l}\text { Desempeños claves asociados } \\
\text { con la producción }(\mathrm{DCP})\end{array}$ \\
\hline Mercadeo & $\begin{array}{l}\text { Desempeños claves asociados } \\
\text { con el mercadeo }(\mathrm{DCM})\end{array}$ \\
\hline Innovación & $\begin{array}{l}\text { Desempeños claves asociados } \\
\text { con la innovación }(\mathrm{DCI})\end{array}$ \\
\hline
\end{tabular}

La identificación exige, además del conocimiento profundo de la organización por parte de su propietario/administrador o equipo directivo, el concurso de empleados y trabajadores conocedores de las rutinas de la organización. Asimismo, se sugiere, en caso de ser necesario, el acompañamiento o asesoría de un experto conocedor de las características y factores de éxito de la industria.

11 Se puede establecer cierta correspondencia entre las competencias básicas del análisis funcional y las actividades primarias y de apoyo de la cadena de valor. Así, por ejemplo, la producción corresponde a las operaciones y logística interna; innovación al desarrollo tecnológico de apoyo y al mercadeo y servicios. 


\subsubsection{Identificación de recursos estratégicos vinculados a los desempeños claves}

En esta segunda etapa, una vez definidas las capacidades básicas y desempeños claves, se procede a relacionar todos los recursos de la empresa con estos últimos. Con este propósito, a cada desempeño clave se asocian los recursos necesarios en su ejecución y la de otro(s) desempeño(s) clave(s). En la práctica, se procede a hacer un listado de los recursos efectivamente disponibles para la empresa y a definir su vínculo específico con los diferentes desempeños claves de la empresa, como se muestra en la tabla 3.

Tabla 3. Recursos empresariales y desempeños claves

\begin{tabular}{c|c|c} 
Producción, & Mercadeo, & Innovación, \\
$D C P_{p},(p=1,2, \ldots, a)$ & $D C M_{m},(m=1,2, \ldots, b)$ & $D C I_{n},(n=1,2, \ldots, c)$
\end{tabular}

Recursos de la empresa asociados con los desempeños claves $\left(R E_{j}\right)$, donde $i=p, m$, y/o $n$, dependiendo del o los desempeños claves a los que se vinculan.

\subsection{Examen del valor estratégico de los recursos}

Se consideran críticos o estratégicos los recursos cuyas propiedades permiten generar, sostener y apropiar la ventaja competitiva, de acuerdo con lo expuesto en sección 3.2.1. Del examen de todos los recursos de la empresa $\left(R E_{i}\right)$ se califican como críticos o estratégicos aquellos que cumplen de manera satisfactoria con los exámenes de superioridad competitiva, facilidad de imitación, durabilidad, apropiabilidad y facilidad de sustitución.

Con propósitos operativos se sugiere la calificación de cada test en una escala numérica de 1 a 10 , en la cual el rango menor que 5 corresponde a una evaluación de "baja", de 5.1 a 7.5, "media" y mayor que 7.6 "alta"; se consideran críticos o estratégicos aquellos recursos con una calificación "media" o "alta" (mayor que 7.5 en la calificación promedio). En la tabla 4 se presenta el esquema de registro de la valoración del recurso, se sugiere adicionar a la valoración cualitativa una justificación verbal.

Tabla 4. Prueba del valor estratégico del recurso

\begin{tabular}{|c|c|c|}
\hline \multicolumn{3}{|c|}{ Identificación del recurso: $R E_{i}$} \\
\hline TEST & $\begin{array}{l}\text { Valoración } \\
\text { cuantitativa } \\
(1 \text { a } 10)\end{array}$ & Justificación \\
\hline \multicolumn{3}{|l|}{$\begin{array}{l}\text { Superioridad } \\
\text { competitiva }\end{array}$} \\
\hline \multicolumn{3}{|l|}{ Imitabilidad } \\
\hline \multicolumn{3}{|l|}{ Durabilidad } \\
\hline \multicolumn{3}{|l|}{ Apropiabiliadad } \\
\hline \multicolumn{3}{|l|}{ Facilidad de sustitución } \\
\hline $\begin{array}{c}\text { VALOR } \\
\text { ESTRATÉGICO (VE) }\end{array}$ & $\begin{array}{l}\mathrm{VE}=\text { valoración } \\
\text { cuantitativa promedio }\end{array}$ & \\
\hline
\end{tabular}

Fuente: elaboración propia a partir de Rangote (1999, p. 239).

\subsection{Evaluar la consistencia estratégica de los recursos}

El valor estratégico del recurso mide su habilidad para generar y sostener la ventaja competitiva de la empresa en el largo plazo, pero no informa sobre su habilidad de contribuir con el propósito estratégico; esta última se denomina 'consistencia estratégica' y, sustancialmente, mide el alineamiento del recurso con el propósito estratégico de la empresa (Rangone, 1999, p. 240).

Como principio de valoración de la consistencia estratégica se plantea que el propósito último no es atraer el mayor número de clientes, sino la obtención de mayores beneficios a través de una ventaja competitiva sostenible asociada, a su vez, con las características estratégicas de 
recursos y capacidades. La medición de la consistencia estratégica se sigue en tres pasos, a saber:

1. Determinar la importancia relativa de los desempeños claves asociados con cada capacidad y, para estas últimas, determinar su prioridad relativa. Esta valoración cualitativa, en la escala bajo, medio, alto responde a la pregunta ¿cuál es la importancia del recurso o capacidad en conferir la ventaja competitiva sustentable?

2. Determinar el impacto de cada recurso en el desempeño clave relevante (recuérdese que un recurso puede impactar más de un desempeño).

3. La consistencia estratégica del recurso resulta de la integración de las medidas de las prioridades relativas de la capacidad y el desempeño clave (paso 1) con el impacto del recurso en el desempeño (paso 2).

La valoración final de la consistencia estratégica del recurso se hace en una escala cualitativa de valoración que incluye los valores bajo, medio y alto, definidos por las calificaciones alcanzadas en los pasos 1 y $2^{12}$.

12 Grant (2010, p. 144) incluye, como parte de la consistencia estratégica, una valoración de lo que denomina 'la fortaleza relativa del recurso'. No obstante, advierte sobre las dificultades propias del ejercicio de benchmarking requerido para estas valoraciones, el cual incluye la identificación, comprensión y adopción de las prácticas más sobresalientes de las organizaciones en cualquier parte del mundo. Por esa razón no se incluyen en el método presentado en este trabajo.

\subsection{Definición de opciones estratégicas de la empresa}

A partir del valor estratégico (VE) y la consistencia estratégica $(\mathrm{CE})$ de los recursos se determinan las opciones estratégicas de la empresa. Este análisis se sintetiza en la matriz de la tabla 5 que asigna los recursos en cuatro casillas:

Tabla 5. Matriz de valores y consistencia estratégica de los recursos

\begin{tabular}{|c|c|c|c|}
\hline VE & $\mathrm{CE}$ & Baja & Alta \\
\hline & Bajo & No significativo & II \\
\hline & Alto & III & I \\
\hline
\end{tabular}

Fuente: Rangone (1999, p. 241).

- Cuadrante I: recursos de alta consistencia estratégica (CE) y valor estratégico (VE), coherentes con los objetivos estratégicos de la empresa y capaces de generar ventaja competitiva.

- Cuadrante II: recursos de alta consistencia estratégica $(\mathrm{CE})$ pero bajo valor estratégico (VE), alineados con los objetivos estratégicos pero incapaces de generar ventaja competitiva de largo plazo.

- Cuadrante III: recursos de baja consistencia estratégica $(\mathrm{CE})$ pero de alto valor estratégico $(\mathrm{VE})$, recursos que, aunque críticos o estratégicos, no se encuentran alineados con el objetivo estratégico de la empresa.

- No significativo: recursos de baja consistencia estratégica $(\mathrm{CE})$ y bajo valor estratégico $(\mathrm{VE})$. 
Del análisis detallado de la matriz, el equipo de dirección o el propietario administrador podrán deducir las guías para la formulación de la estrategia de la empresa en dos niveles fundamentales:

1. Alineación global entre los objetivos estratégicos de la empresa y sus recursos críticos. La consistencia estratégica existe si la mayoría de los recursos se encuentran en los cuadrantes I y II; sin embargo, si muchos recursos se encuentran en el cuadrante III, existe una inconsistencia significativa que debe conllevar a la revisión de los propósitos estratégicos de la empresa en relación con los factores claves de éxito en la industria.

2. Las estrategias más accesibles para diferentes clases de recursos basadas en los siguientes criterios:

- Recursos en el cuadrante I: deberían ser explotados en tanto que sirven de soporte a los desempeños claves y, al mismo tiempo, fortalecidos para evitar pérdida de valor.

- Recursos en el cuadrante II: deberían ser desarrollados mediante inversión con el fin de incrementar su valor.

- Recursos en el cuadrante III: deberían ser explotados sin invertir en ellos y, en la medida de lo posible, conducidos a explotar otros desempeños claves.

En todos los casos, en la formulación de la empresa se bebe tener presente que los recursos de la empresa pueden ser potenciados de las siguientes maneras (Grant, 2010, p. 149):
- Concentración mediante procesos de convergencia en torno a metas definidas y consistentes.

- Acumulación para facilitar el aprendizaje.

- Complementación para incrementar su efectividad mediante el vínculo con otros recursos y capacidades de la empresa.

- Conservación y reciclaje mediante el uso de capacidades de la empresa.

- Cooptación a través de acuerdos con otras empresas.

Asimismo, las capacidades de la empresa pueden ser replicadas y creadas internamente; una vez la capacidad ha sido creada, su réplica es menos costosa, por lo menos en los casos en los que el conocimiento implicado es codificable, con base en la sistematización del conocimiento subyacente mediante la formulación de procedimientos estandarizados de operación. No obstante, la mayor dificultad para la réplica de capacidades radica en que la sistematización del conocimiento supone que la empresa está en capacidad de articular completamente los procesos (actividades) que las soportan.

Por su parte, la creación de recursos y capacidades, además de demandar tiempo, puede ser difícil y costosa, lo cual no obsta para que la organización no lo intente. La pregunta clave por responder es ¿qué puede hacer la organización en un tiempo limitado para apropiar las rutinas que subyacen a las capacidades?; estas involucran grupos de recursos operando en equipo para alcanzar un cierto nivel de desempeño, cuya dinámica se conoce de manera esquemática en la mayoría de los casos. 


\section{Referencias}

Amit, R. \& and Schoemaker, P. (1993). Strategic Assets and Organizational Rent Strategic Management Fournal, 14(1), 33-46.

Anzola, O. y Puentes, M. (2006). Determinantes de las acciones gerenciales. En Microempresas y Empresas Pymes. Bogotá: Universidad Externado de Colombia.

Aragón, A. y Sánchez, G. (agosto-septiembre, 2002). Orientación estratégica, características de gestión y resultados: un estudio en las pymes españolas. Tribuna de Economía, No. 809, 169-188. Información Comercial Española - ICE.

Ayala Ruiz, L. (2011). Gerencia de Mercadeo. Las estrategias competitivas genéricas de Porter. (Apuntes de Clase).[http://www.3w3search.com/Edu/Merc/Es/ GMerc057.htm].

Baena, E., Botero, C. y Montoya, O. (julio, 2003). Gestión tecnológica y competitividad. Scientia et Technica, No. 21, 121-126.

Beltrán, A., Torres, E. y Bello, C. (2004). Pymes: un reto a la competitividad. Bogotá: Universidad Externado de Colombia.

Beltrán, A., Torres, E. y Bello, C., Anzola, O. y Camargo, R. (2005). Mejorando la competitividad de la pyme, desarrollo y aplicación de modelos de gestión. Bogotá: Universidad Externado de Colombia.

Benzaquen, J., del Carpio L. A., Valdivia, C. y Zegarra, L. (diciembre, 2010). Un índice regional de competitividad para un país. Revista de la CEPAL, 102, 69-86.

Casablanca, C. (2001). Recursos y capacidades, factores institucionales y resultados de las universidades públicas catalanas: una propuesta de marco conceptualy exploración de sus constructos. Universidad Autónoma de Barcelona, Doctorado en Creación, Estrategia y Gestión de Empresas [Tesis doctoral].

Garelli, S. (2006). The competitiveness of nations: the fundamentals. IMD World competitivenes Tearbook 2006. [http://www.imd.ch/documents/wcc/content/ Fundamentals.pdf]

González, O. y González, J. (2011). El sector de Artes Gráficas en Bucaramanga y su área metropolitana. Un análisis de competitividad y productividad. En Modernización e innovación para la gestión de las Pymes: conceptos, herramientas y casos. Cap. III, Bogotá, Redpymes.

Grant, R. M. (1991). The Resource-Based Theory of Competitive Advantage: Implications for Strategy Formulation, California Management Review, 114-35.

Grant, R. M. (2010). Contemporary Strategy Analysis, Sevent Edition, (Chapter 5. Analyzing Resources and Capabilities), (pp.123-168). http://www.blackwellpublishing.com/grant/files/CSAC05.pdf [7/03/2012, 3:30 p.m.].

Helfat C., E. \& Lieberman, M. (2002). The Birth of Capabilities: Market Entry and the Importance of Prehistory. Industrial and Corporate Change, 11, 725-60.

Kantis, H. (1996). Inercia e innovación en las conductas estratégicas de las pymes argentinas. Documento de Trabajo, 73, CEPAL, Buenos Aires.

López Ortega, E. (s.f.). Modelo dinámico para evaluar la competitividad regional. Instituto de Ingeniería - UNAM. 
Mintzberg, H. \& Waters, J. A. (1985). Of Strategies, Deliberate and Emergent. Strategic Management Fournal, 6, 257-72.

Moreno, N. (2011). Análisis situacional empresa ABC. En Modernización e innovación para la gestión de las pymes: conceptos, herramientas y casos. Cap. II, Bogotá, Redpymes.

Nelson R. R. \& Winter, S. G. (1982). An Evolutionary Theory of Economic Change. Cambridge, MA, Belknap.

Observatorio del Caribe Colombiano (OCG), Cámara de Comercio de Cartagena (CCG). (2009). Perfil competitivo de las empresas turísticas de Cartagena. Serie de estudios sobre la competitividad de Cartagena, No. 15.

O'Regan, N. \& Ghobadian, A. (2002). Effective strategic planning in small and medium sized firms, Management Decision, 40(7), 663-671.

Pérez Uribe, R. et ál. (2009). Modelo de modernización para la gestión de organizaciones. Universidad EAN.

Peterlaf, M. A. (1993). The Cornerstones of Competitive Advantage: A Resource-Based View, Strategic Management fournal, 14, 179-92.

Porter, M. (1985). Competitive Advantage. The Free Press, New York.

Porter, M. (1991). La ventaja competitiva de las naciones. Buenos Aires: Javier Vergara Editor.

Porter, M. (nov-dec, 1996). What is strategy?, Harvard Business Review, 61-78.

Porter, M. (2000). Estrategia competitiva: técnicas para el análisis de los sectores industriales y de la competencia. México, D.F.: Compañía Editorial Continental.
Prahalad, G.K. \& Hamel, G. (may-june, 1990). The Core Competence of Corporation, Harvard Business Review, $1-15$.

Ramírez, M. (2011). El MMGO y la planeación estratégica. Aplicación a dos casos empresariales. En Modernización e innovación para la gestión de las pymes: conceptos, herramientas y casos. Cap. IV, Bogotá, Redpymes.

Rangone, A. (1997). Linking Organisational Effectiveness, Key Success Factors and Performance Measures: An Analytical Framework. Management Accounting Research, 8, 207-219.

Rangone, A. (1999). A resource-based approach to strategy analysis in small medium sized enterpraises. Small Busines Economy, 12, 233-248.

Rangone, A. (1998). On the Applicability of Analytical Techniques for the Selection of AMTs in SmallMedium Sized Firms. Small Business Economics, 10, 293-304.

Ryan, M. (2003). Towards enabling competitive advantage in small-to-medium enterprises, School of Computing. Dublin Institute of Technology, Ireland [http://www.comp. dit.ie/rfitzpatrick/MSc_Publications/2003_Maria_ Ryan.pdf].

Stonehouse, G. \& Pemberton, J. (2002). Strategic Planning in SMEs - some empirical findings. Management Decision, 40(9), 853-861.

Williamson, O. (1975). Mercados y jerarquías; su análisis y sus implicaciones antitrust. México: Fondo de Gultura Económico. 